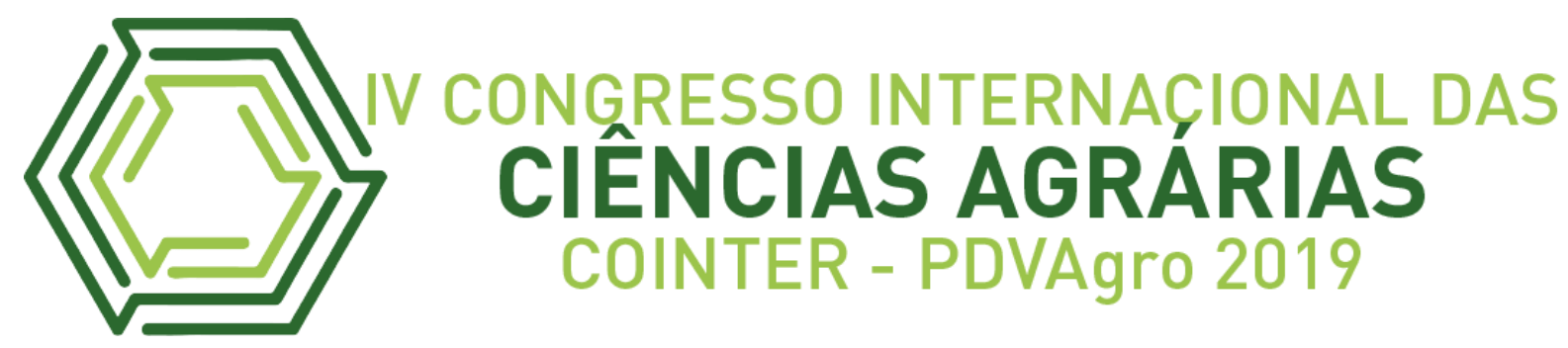

\title{
AVALIAÇÃO DA ATIVIDADE ANTIOXIDANTE DE FARINHAS DA CASCA DE MANGA SUBMETIDA A PROCESSOS DE CONSERVAÇÃO PELA ADIÇÃO DE INIBIDORES DE REAÇÃO E REFRIGERAÇÃO
}

\section{EVALUATION OF ANTIOXIDATING ACTIVITY OF MANGO SHELL FLOURS SUBMITTED FOR CONSERVATION PROCESSES BY THE ADDITION OF REACTION INHIBITORS AND COOLING}

\section{EVALUACIÓN DE LA ACTIVIDAD ANTIOXIDANTE DE HARINA DE cáscara MANGO PRESENTADA PARA PROCESOS DE CONSERVACIÓN POR LA ADICIÓN DE INHIBIDORES DE REACCIÓN Y REFRIGERACIÓN}

\author{
Apresentação: Comunicação Oral \\ Cinara Vanessa de Muniz Almeida ${ }^{1}$; Dayane Nunes Barros²; José Renato da Silva ${ }^{3}$; Rodrigo
} Lira de Oliveira ${ }^{4}$; Suzana Pedroza da Silva ${ }^{5}$

\section{DOI: https://doi.org/10.31692/2526-7701.IVCOINTERPDVAgro.2019.0079}

\section{Resumo}

A casca da manga apresenta características nutricionais importantes para o aproveitamento como complementação alimentar, por exemplo, na forma de farinhas. Porém, as cascas devem passar por processos de conservação para evitar perecividade e aumentar sua vida útil. As principais características verificadas na casca da manga são suas propriedades antioxidantes, uma vez que estas apresentam benefícios à saúde do consumidor, logo verificar desenvolvimento de novos produtos com maior valor nutricional agregado é uma das necessidades atuais da indústria de alimentos. Com isto o presente estudo tem como objetivo avaliar a atividade antioxidante da farinha da casca de manga submetida a processos de conservação pela adição de inibidores de reação tais como o ácido ascórbico e o cloreto de cálcio e, utilização de refrigeração As mangas foram adquiridas no comércio local de Garanhuns-PE. Foram lavadas, sanitizadas, descascadas, removida a polpa, homogeneizadas e separadas em seis grupos: controle (sem tratamento) e quatro com tratamentos (A (1,0\% (AA) + 1,0\% $\left.\left(\mathrm{CaCl}_{2}\right)\right)$; B $\left(2,0 \%(\mathrm{AA})+2,0 \%\left(\mathrm{CaCl}_{2}\right)\right) ; \mathrm{C}(2,0 \%(\mathrm{AA})$ $+1,0 \%\left(\mathrm{CaCl}_{2}\right) \mathrm{D}\left(1,5 \%(\mathrm{AA})+1,5 \%\left(\mathrm{CaCl}_{2}\right)\right)$. As cascas foram acondicionadas em embalagens de polietileno e armazenadas sob refrigeração $\left(2{ }^{\circ} \mathrm{C}, 5{ }^{\circ} \mathrm{C}\right.$ e $\left.7{ }^{\circ} \mathrm{C}\right)$. Com análise dos componentes principais foram determinados os dois melhores tratamentos então foram produzidas farinhas a partir destas através da secagem em estufa a $60{ }^{\circ} \mathrm{C} / 24 \mathrm{~h}$ e realizaram-

\footnotetext{
${ }^{1}$ Graduanda em Engenharia de Alimentos, UFRPE, Garanhuns, cinaravanessa76@gmail.com

${ }^{2}$ Graduanda em Engenharia de Alimentos, UFRPE, Garanhuns, dayanenunes1811@outlook.com

${ }^{3}$ Graduanda em Engenharia de Alimentos, UFRPE, Garanhuns, j_renatosilva123@hotmail.com

${ }^{4}$ Doutorando em Biotecnologia- RENORBIO, UFRPE, Garanhuns, rodrigolira1@outlook.com

${ }^{5}$ Doutora em Engenharia Química, UFRPE, Garanhuns, suzpedroza@gmail.com
} 
se as análises de atividade antioxidante ABTS (\%), atividade antioxidante DPPH (\%), Compostos Fenólicos $(\mathrm{mg} / 100 \mathrm{~g})$ e flavonóides $(\mathrm{mgEQ} / 100 \mathrm{~g})$. A farinha proveniente do tratamento B apresentou maiores teores de: atividade antioxidante DPPH $(86,827 \pm 0,0103 \%)$, compostos fenólicos $(337,281 \pm 1,105 \mathrm{mgEQ} / 100 \mathrm{~g})$ e flavonoides $(39,306 \pm 0,575 \mathrm{mgEQ} / 100 \mathrm{~g})$; o maior teor de atividade antioxidante ABTS foi obtido para amostra submetida ao tratamento D $(93,451 \pm 1,001 \%)$. As farinhas de casca de manga apresentaram potencial antioxidante, com destaque para farinha proveniente do tratamento B, o emprego dos tratamentos com inibidores de reação (ácido ascórbico e cloreto de cálcio) indicando maior estabilidade de armazenamento e preservação de compostos antioxidantes em relação a farinha não submetida a adição de inibidores.

Palavras-Chave: ABTS, Compostos fenólicos, DPPH, flavonoides.

\section{Resumen}

La cáscara de mango tiene características nutricionales importantes para su uso como complemento alimenticio, por ejemplo en forma de harina. Sin embargo, los depósitos deben someterse a procesos de conservación para evitar la pericidad y aumentar su vida útil. Las principales características que se encuentran en la cáscara de mango son sus propiedades antioxidantes, ya que tienen beneficios para la salud del consumidor, por lo que verificar el desarrollo de nuevos productos con mayor valor nutricional agregado es una de las necesidades actuales de la industria alimentaria. Por lo tanto, el presente estudio tiene como objetivo evaluar la actividad antioxidante de la harina de cáscara de mango sometida a procesos de conservación mediante la adición de inhibidores de reacción como el ácido ascórbico y el cloruro de calcio y la utilización de refrigeración. Sitio de Garanhuns-PE. Fueron lavados, desinfectados, pelados, quitados de pulpa, homogeneizados y separados en seis grupos: control (sin tratamiento) y cuatro con tratamientos (A $(1.0 \%$ (AA) $+1.0 \%$ $(\mathrm{CaCl} 2))$; $\mathrm{B}(21.0 \%(\mathrm{AA})+2.0 \%(\mathrm{CaCl} 2)) \mathrm{C}(2.0 \%(\mathrm{AA})+1.0 \%(\mathrm{CaCl} 2) \mathrm{D}(1.5 \%(\mathrm{AA})+$ $1.5 \%(\mathrm{CaCl} 2)$ Los depósitos se envasaron en recipientes de polietileno y se almacenaron en refrigeración $\left(2^{\circ} \mathrm{C}, 5^{\circ} \mathrm{C}\right.$ y $\left.7^{\circ} \mathrm{C}\right)$. Con el análisis de los componentes principales, se produjeron los dos mejores tratamientos. $60^{\circ} \mathrm{C} / 24$ hy se realizaron los siguientes análisis: actividad antioxidante ABTS (\%), actividad antioxidante DPPH (\%), compuestos fenólicos (mg / 100g) y flavonoides (mgEQ / 100g). mayor contenido de: actividad antioxidante DPPH $(86.827 \pm 0.0103 \%)$, compuestos fenólicos $(337.281 \pm 1.105 \mathrm{mgEQ} / 100 \mathrm{~g})$ y flavonoides $(39.306 \pm 0.575 \mathrm{mgEQ} / 100 \mathrm{~g})$, la mayor actividad antioxidante ABTS se obtuvo para la muestra s sometido a tratamiento D $(93.451 \pm 1.001 \%)$. Las harinas de cáscara de mango mostraron potencial antioxidante, especialmente la harina del tratamiento $\mathrm{B}$, el uso de tratamientos inhibidores de la reacción (ácido ascórbico y cloruro de calcio) indicando una mayor estabilidad de almacenamiento y preservación de compuestos antioxidantes en relación con la no harina. sometido a la adición de inhibidores..

Palabras Clave: ABTS, Compuestos fenólicos, DPPH, flavonoides.

\footnotetext{
Abstract

Mango peel has important nutritional characteristics for use as a food supplement, for example in the form of flour. However, the shells must undergo conservation processes to avoid pericity and increase their shelf life. The main characteristics found in mango peel are
} 
its antioxidant properties, since these have benefits to the health of the consumer, so verifying the development of new products with higher added nutritional value is one of the current needs of the food industry. Thus the present study aims to evaluate the antioxidant activity of mango peel flour subjected to preservation processes by the addition of reaction inhibitors such as ascorbic acid and calcium chloride and refrigeration utilization. Garanhuns-PE site. They were washed, sanitized, peeled, pulp removed, homogenized and separated into six groups: control (no treatment) and four with treatments (A (1.0\% (AA) + 1.0\% (CaCl2)); B $(2$ $1.0 \%(\mathrm{AA})+2.0 \%(\mathrm{CaCl} 2)) \mathrm{C}(2.0 \%(\mathrm{AA})+1.0 \%(\mathrm{CaCl} 2) \mathrm{D}(1.5 \%(\mathrm{AA})+1.5 \%(\mathrm{CaCl} 2)$ The shells were packed in polyethylene containers and stored under refrigeration $\left(2{ }^{\circ} \mathrm{C}, 5^{\circ} \mathrm{C}\right.$ and $7^{\circ} \mathrm{C}$.) With analysis of the main components, the two best treatments were then produced. $60^{\circ} \mathrm{C} / 24 \mathrm{~h}$ and the following analyzes were performed: ABTS antioxidant activity (\%), DPPH antioxidant activity (\%), phenolic compounds ( $\mathrm{mg} / 100 \mathrm{~g}$ ) and flavonoids (mgEQ / 100g). highest content of: DPPH antioxidant activity $(86.827 \pm 0.0103 \%)$, phenolic compounds $(337.281 \pm 1.105 \mathrm{mgEQ} / 100 \mathrm{~g})$ and flavonoids $(39.306 \pm 0.575 \mathrm{mgEQ} / 100 \mathrm{~g})$, the highest ABTS antioxidant activity was obtained for sample s subjected to treatment D $(93.451 \pm 1.001 \%)$. Mango peel flours showed antioxidant potential, especially flour from treatment $\mathrm{B}$, the use of reaction inhibitor treatments (ascorbic acid and calcium chloride) indicating greater storage stability and preservation of antioxidant compounds in relation to non-flour. subjected to the addition of inhibitors.

Keywords: ABTS,Phenolic compounds, DPPH, flavonoids.

\section{Introdução}

Os antioxidantes desempenham funções importantes em alimentos industrializados e podem ocasionar benefícios ou malefícios ao organismo humano, em decorrência da proporção de consumo e da fonte de obtenção do antioxidante, sendo os antioxidantes naturais os mais benéficos ao organismo humano resultando em iterações positivas a saúde do consumidor.

As frutas e hortaliças são muito valorizadas devido a altos teores de antioxidantes, entre os resíduos provenientes de frutas as cascas de frutas é o mais rico em teores de antioxidantes sendo os principais: compostos polifenólicos, flavonoides e ácido ascórbico, isto torna-os valiosos e de interesse da área alimentícia com excelente capacidade antioxidante e propriedades funcionais. Os compostos fenólicos são um tipo de antioxidante natural, com destaque para os flavanoides, os mais significativos do grupo.

O Brasil é um dos maiores produtores de frutas e entre estas está a manga (Mangífera indica L.), rica em diversos nutrientes, tanto na polpa quanto na casca, porem o processamento de frutas por vezes resulta na eliminação de partes consideradas não comestíveis que apresentam potencial de ser aplicada em dietas e significativas propriedades 
nutracênicas a alimentação, além de reduzir os impactos ambientais decorrentes dos resíduos orgânicos provenientes das indústrias de processamento de frutas.

A casca de manga apresenta propriedades, muito interessantes para o enriquecimento de dietas por exemplo poderia ser incorporada a alimentação na forma de farinha, visto que o consumo de farinhas é altamente significativo na dieta dos brasileiros

A casca da manga é altamente perecível e através do processamento dessas cascas na forma de farinhas seriam atreladas a estas vantagens como redução da perfectibilidade, maior tempo hábil para consumo, praticidade além do valor agregado a mesma

Por vezes o processamento destas cascas de manga não pode ocorrer de maneira imediata sendo assim necessária a utilização de métodos de conservação até que ocorra o processamento, uma vez que as cascas de frutas sofrem com problemas de escurecimento oxidativo, perda de massa, desenvolvimento de microrganismos e atuação de enzimas.

Diversos métodos de conservação podem ser empregados com a finalidade de inibir reações indesejáveis, assim como manter as características adequadas para o consumo, porém, a depender da finalidade a que estas se destinam alguns métodos se tornam mais adequados, como o emprego de refrigeração, e a aplicação de soluções visando reduzir reações desfavoráveis a conservação da casca, visto que se trata de um tecido vegetal.

O emprego de soluções inibidoras de reação é um método de conservação muito interessante para ser aplicado a tecidos vegetais, como cascas de frutas, pois irão desempenhar funções importantes na manutenção das características e qualidade da casca da manga.

Dentre as substâncias empregadas na conservação de frutas e hortaliças temos o cloreto de cálcio e o ácido ascórbico, o cálcio presente no cloreto de cálcio interage com a pectina presente na parede celular do alimento, formando o pectato de cálcio proporcionando melhoria na firmeza e textura, já o ácido ascórbico pelo seu poder antioxidante, atua inibindo reações oxidativas e prevenindo o escurecimento enzimático.

Neste contexto, a indústria de alimentos tem buscado produtos e processos, onde as características funcionais dos alimentos sejam conservadas na expectativa de atender um público mais exigente, informado e consciente. Na perspectiva de melhoria deste panorama, pesquisas científicas tornam-se necessárias no desenvolvimento de novos produtos com maior valor nutricional agregado, com isto o presente estudo objetiva avaliar a atividade antioxidante da farinha da casca de manga submetida a processos de conservação pela adição 
de inibidores de reação tais como o ácido ascórbico e o cloreto de cálcio e, utilização de refrigeração.

\section{Fundamentação Teórica}

Um antioxidante se define como uma substância que, em baixas concentrações, retarda ou previne a oxidação do substrato, é considerado antioxidante a substância que possui substituintes doadores de elétrons ou de hidrogênio ao radical. Os antioxidantes são classificados quanto a inibição de radicais livres, podendo ser primários ou biológicos, naturais ou sintéticos (MORAIS et al., 2013).

Os compostos antioxidantes artificiais, tais como hidroxil-tolueno-butilado (BHT) e butil-hidroxi-anisol (BHA) são comumente utilizados em alimentos processados, porém alguns estudos têm relatado que estes compostos apresentam alguns efeitos colaterais e podem ser cancerígenos (EL-FAHAM et al., 2016)

Estudos indicam que o consumo de alimentos ricos em antioxidantes naturais está associado com a baixa incidência de doenças degenerativas, incluindo o câncer, doenças cardiovasculares, inflamações e artrites. As frutas e os vegetais apresentam em sua composição muitos compostos antioxidantes naturais, tais como compostos fenólicos, carotenóides e antocianinas, isto têm atraído grande interesse por parte das indústrias, dos consumidores e da comunidade científica. Dentre as várias partes que compõe as frutas e os vegetais, as cascas de frutos se destacam, devido a capacidade antioxidante e propriedades funcionais (YU-GE-LU; FEI-YUE; QIONG, 2017; EL-FAHAM et al., 2016).

Os compostos fenólicos são um tipo de antioxidante natural, mais ativo em vegetais. (SUCUPIRA et al., 2012). Pertencente a classe dos fitoquímicos alimentares, podem se ligar a radicais livres eliminando-os (SOUSA et al., 2016). Vários compostos fenólicos obtidos a partir de fontes vegetais, tais como frutos, casca, folhas e raízes atuam contra o câncer (MASUD PARVEZ; MOSADDIK, 2016).

O Brasil é um dos maiores produtores de frutas e entre estas está a manga (Mangífera indica L.), que é uma fruta tropical, que possui sabor agradável e características exóticas, devido a isto apresenta altos índices de aceitabilidade por parte dos consumidores (MORENO, 2016).

$\mathrm{Na}$ indústria de processamento de alimentos após o uso da polpa das frutas grande parte das vezes ocorre o descarte dos resíduos como cascas e sementes, estas por sua vez 
podem apresentar nutrientes que poderiam ser utilizados na fortificação alimentar ou na composição de alimentos (MORENO, 2016).

As cascas são o mais importante subproduto do processamento de manga, corresponde a aproximadamente $7-24 \%$ do fruto. Uma vez que estes resíduos podem apresentar potencial de ser aplicados na alimentação e são realizados estudos na tentativa de utilizar eficientemente a casca de manga na alimentação humana (EL-FAHAM et al., 2016)

Dentre as variedades de mangas, a Tommy Atkins é a mais exportada pelo Brasil e uma das mais cultivadas, isto se deve a sua boa produtividade, boa capacidade de adaptação a diferentes ambientes de cultivo e por apresentar boa tolerância a doenças (MENDES, 2013).

Do ponto de vista nutricional, a casca da manga apresenta características nutricionais importantes, (PADILHA; BASSO, 2015). Devido a sua perecibilidade as cascas da manga devem passar por processos de conservação a fim de aumentar sua vida útil, seja pela adição de conservantes e inibidores de reações, processos que usam resfriamentos ou congelamentos; utilização de embalagens e atmosferas modificadas; aplicação de biofilmes; métodos de secagem ou ainda a combinação de mais de um destes.

Segundo Lemos et al. (2013) os resíduos provenientes da agroindústria de frutas podem ser melhor aproveitados quando são submetidos a processos de secagem. Os resíduos, cascas de mangas, por exemplo, podem ser transformados em farinhas e com isso poderão ser utilizados como ingredientes na produção de diferentes alimentos: bebidas, sobremesas, biscoitos, massas e pães, além da praticidade e da redução de perecibilidade em comparação com cascas de manga in natura (REINOSO et al., 2017).

As farinhas são presentes na dieta do brasileiro e por isso de grande importância, de acordo com resolução de diretoria colegiada - RDC No 263, de 22 de setembro de 2005 da ANVISA farinhas são "os produtos obtidos de partes comestíveis de uma ou mais espécies de cereais, leguminosas, frutos, sementes, tubérculos e rizomas por moagem e/ou outros processos tecnológicos considerados seguros para produção de alimentos."

Por vezes o processamento destas cascas de manga não pode ocorrer de maneira imediata sendo assim necessária a utilização de métodos de conservação até que ocorra o processamento, segundo Miguel (2008) até o processamento, as cascas de frutas sofrem com problemas de escurecimento oxidativo, perda de massa, desenvolvimento de microrganismos e atuação de enzimas. 
A utilização de substâncias como cloreto de cálcio e ácido ascórbico apresentam funções importantes no que se trata de conservação de características de frutas, pois o cálcio presente no cloreto de cálcio interage com a pectina presente na parede celular do alimento formando o pectato de cálcio proporcionando firmeza e melhora na textura dos mesmos e; o ácido ascórbico pelo seu poder antioxidante, muito usado em frutas, vegetais e hortaliças inibindo reações oxidativas e prevenindo o escurecimento enzimático (MIGUEL, 2008).

\section{Metodologia \\ OBTENÇÃO E PREPARO DAS AMOSTRAS}

As mangas foram adquiridas no comércio local de Garanhuns-PE, foram lavadas em água corrente e imersas em solução de hipoclorito de sódio 100 ppm durante 30 minutos para sanitização. As mangas foram descascadas manualmente com faca de aço inoxidável em cortes espiral (FIGURA 1) e removida toda a polpa remanescente, foram acondicionadas em uma bandeja retangular de plástico e mescladas para homogeneização da amostra.

Figura 1. Corte em espiral da casca da manga Tommy Atkins obtida para a produção de manga minimamente

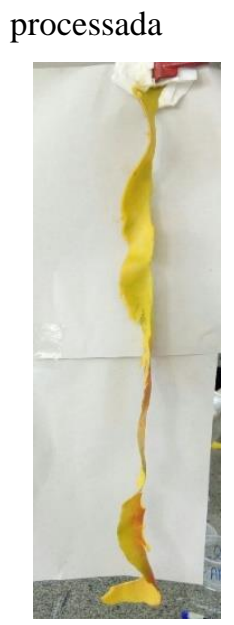

Fonte: Própria (2018).

\section{APLICAÇÃO DAS SOLUÇÕES DE ÁCIDO ASCÓRBICO (AA) E CLORETO DE CÁLCIO $\left(\mathrm{CACL}_{2}\right)$ SOB DIFERENTES TEMPERATURAS}

As cascas de manga foram imersas na solução com AA por 5 minutos, peneiradas e posteriormente imersas na solução de $\mathrm{CaCl}_{2}$ por $5 \mathrm{~min}$, de acordo com as concentrações descritas na Tabela 1. 
Tabela 1 - Tipos de tratamentos realizados no processamento mínimo das cascas de manga

\begin{tabular}{c|c}
\hline Identificação do tratamento & Tratamento \\
\hline Controle & sem AA e $\mathrm{CaCl}_{2}$ \\
A & $1,0 \%(\mathrm{AA})+1,0 \%\left(\mathrm{CaCl}_{2}\right)$ \\
B & $2,0 \%(\mathrm{AA})+2,0 \%\left(\mathrm{CaCl}_{2}\right)$ \\
C & $2,0 \%(\mathrm{AA})+1,0 \%\left(\mathrm{CaCl}_{2}\right)$ \\
D & $1,5 \%(\mathrm{AA})+1,5 \%\left(\mathrm{CaCl}_{2}\right)$ \\
\hline
\end{tabular}

Fonte: Própria (2018).

Após a segunda imersão as cascas foram peneiradas e pesadas (aproximadamente 50 g) para acondicionamento em recipientes de polietileno, transparentes, com tampa sob refrigeração $\left(2{ }^{\circ} \mathrm{C}, 5^{\circ} \mathrm{C}\right.$ e $\left.7{ }^{\circ} \mathrm{C}\right)$, para posterior realização das análises físico-químicas a cada $48 \mathrm{~h}$ durante 8 dias cada tratamento. Todos os tratamentos foram testados nas três temperaturas para melhor determinação da condição ótima de processamento mínimo das cascas de mangas (TABELA 2).

Tabela 2 - Experimentos realizados no processamento mínimo das cascas de manga sob diferentes temperaturas e tratamentos com conservantes

\begin{tabular}{c|c}
\hline Temperatura $\left({ }^{\circ} \mathrm{C}\right)$ & Tratamento \\
\hline 2 & Controle \\
2 & $\mathrm{~A}$ \\
2 & $\mathrm{~B}$ \\
2 & $\mathrm{C}$ \\
2 & $\mathrm{D}$ \\
5 & Controle \\
5 & $\mathrm{~A}$ \\
5 & $\mathrm{~B}$ \\
5 & $\mathrm{C}$ \\
5 & $\mathrm{D}$ \\
7 & Controle \\
7 & $\mathrm{~A}$ \\
7 & $\mathrm{~B}$ \\
7 & $\mathrm{C}$ \\
7 & $\mathrm{D}$ \\
\hline
\end{tabular}

Fonte: Própria (2018). 
Realizaram-se as análises físico-químicas de: teor de umidade (\%), perda de massa fresca $(\%)$, sólidos solúveis totais $\left({ }^{\circ} \mathrm{Brix}\right), \mathrm{pH}$, acidez total titulável (g de ácido cítrico/100 mL de amostra), atividade de água, açúcares $(\mathrm{g} / \mathrm{mL})$, teor de lipídios $(\%)$, teor de fibras $(\%)$ e teor de proteínas (\%); cor e índice de escurecimento. Todas as análises foram realizadas em triplicata a cada 48 horas, de acordo com as metodologias descritas por Detmann et al. (2012), Olivas, Mattinson e Barbosa-cánovas (2007), Maldonade, Carvalho e Ferreira (2013) e Instituto Adolfo Lutz (2008), nos Laboratórios de Análise de Alimentos (LAAL), Laboratório de Nutrição Animal (LANA) e na Central de Laboratórios de Apoio à Pesquisa da Unidade Acadêmica de Garanhuns (CENLAG), ambos na Unidade Acadêmica de Garanhuns da Universidade Federal Rural de Pernambuco (UFRPE/UAG).

\section{ANÁLISE DOS COMPONENTES PRINCIPAIS (ACP)}

Os resultados das análises físico-químicas foram submetidos à análise de componentes principais a partir da matriz de correlação de Pearson, utilizando-se o software XLSTAT 2014.

A partir da ACP dos tratamentos e da amostra controle em todas as temperaturas, selecionaram-se os dois tratamentos que apresentaram melhores resultados.

\section{PRODUÇÃO DA FARINHA DA CASCA DE MANGA TOMMY ATKINS}

Após a avaliação das condições de tratamento para conservação das cascas das mangas, da ACP para determinação das melhores condições, a partir das análises físicoquímicas foi produzida a farinha dentro das condições do tratamento B a $7{ }^{\circ} \mathrm{C}$, do tratamento D a $7{ }^{\circ} \mathrm{C}$ e da condição controle a $7{ }^{\circ} \mathrm{C}$. As cascas após passarem pelo processo de conservação, durante 192 horas foram secas em estufa (FANEM® 515, SÃO PAULOBRASIL) a $60{ }^{\circ} \mathrm{C}$ por 24 horas. Foram trituradas em liquidificador doméstico e peneiradas em peneira de $1 \mathrm{~mm}$. As farinhas foram armazenadas a temperatura ambiente $\left( \pm 25{ }^{\circ} \mathrm{C}\right) \mathrm{em}$ recipientes de polietileno transparente, com tampa, e recoberta com filme de policloreto de polivinila (PVC) para evitar absorção de umidade. Posteriormente foi realizada a caracterização dessas farinhas e realizada nova avaliação. 
Realizaram-se as análises de atividade antioxidante ABTS (\%), atividade antioxidante DPPH (\%), Compostos Fenólicos (mg/100 g) e flavonoides (mgEQ/100 g). Todas as análises foram realizadas em triplicata de acordo com as metodologias descritas por Tseng et al. (2006) com modificações, Li et al. (2008), Vieira (2011), Oliveira (2016), Wolsky e Salatino (1998). Swain e Hills (1959) e Singleton e Rossi (1965) com adaptações, nos Laboratórios de Análise de Alimentos (LAAL) e na Central de Laboratórios de Apoio à Pesquisa da Unidade Acadêmica de Garanhuns (CENLAG), ambos na Unidade Acadêmica de Garanhuns da Universidade Federal Rural de Pernambuco (UFRPE/UAG). Os dados foram interpretados por análise de variância (ANOVA) e Teste de Tukey adotando o nível de significância de 5\%, utilizando o software Minitab 17.

\section{ATIVIDADE ANTIOXIDANTE ABTS}

Realizaram-se as análises de atividade antioxidante ABTS (\%), atividade antioxidante DPPH (\%). Para a determinação da capacidade antioxidante in vitro, pelo método de captura do radical 2,2-azinobis-3-etilbenzotiazolina-6-ácido sulfônico (ABTS). O cátion ABTS• foi formado a partir da reação de soluções aquosas de $7 \mathrm{mM}$ de ABTS e 2,45 $\mathrm{mM}$ de persulfato de potássio $(1: 1 \mathrm{v} / \mathrm{v})$ por $16 \mathrm{~h}$, à temperatura ambiente e na ausência de luz. Após o tempo necessário, a solução radical ABTS foi diluída água para uma absorbância de $0,700( \pm 0,05)$, a $734 \mathrm{~nm}$. Alíquotas de $0,5 \mathrm{~mL}$ da amostra diluída foram adicionadas em tubos de ensaio, juntamente com 3,5 mL da solução do radical ABTS e em seguida a mistura foi lida a $734 \mathrm{~nm}$, após 6 min no escuro. Um controle negativo foi preparado com a utilização e água destilada. Todos os ensaios ocorreram em triplicata.

Todos os ensaios ocorreram em triplicata. A atividade antioxidante foi calculada em relação à atividade de eliminação do radical (\%).

\section{ATIVIDADE ANTIOXIDANE DPPH}

Para a determinação da capacidade antioxidante in vitro, de acordo com o método de captura do radical 1,1-difenil-2-picrilhidrazil (DPPH). A mistura reacional consistiu em 3,5 $\mathrm{mL}$ de DPPH 0,1 mM em metanol e alíquota de $0,5 \mathrm{~mL}$ dos extratos de pigmentos de $\mathrm{M}$. ruber diluídos (quantas vezes necessárias). A absorbância da mistura foi medida a $517 \mathrm{~nm}$ após 30 min de reação no escuro. 
Todos os ensaios ocorreram em triplicata. A atividade antioxidante foi calculada em relação à atividade de eliminação do radical (\%).

\section{DETERMINAÇÃO DE TEOR DE COMPOSTOS FENÓLICOS}

A determinação dos compostos fenólicos totais ocorre empregando o reagente FolinCiocalteu. Em tubos de ensaio serão adicionados $0,5 \mathrm{~mL}$ da solução $(5 \mathrm{~g}$ de farinha:50 $\mathrm{mL}$ de água destilada) e será acrescentado $8,0 \mathrm{~mL}$ de água destilada e $0,5 \mathrm{~mL}$ do reagente de FolinCiocalteau e 1,0 mL de uma solução saturada de carbonato de sódio $\left(\mathrm{Na}_{2} \mathrm{CO}_{3}\right)$ e posteriormente as amostras reacionais foram homogeneizadas em vórtex e mantida em repouso por 60 min na ausência de luz. Transcorrido esse tempo, a mistura foi submetida a leitura em espectrofotômetro em absorbância de $720 \mathrm{~nm}$. Os resultados obtidos serão comparados com uma curva padrão de ácido gálico e serão expressos mgEAG/100g da farinha (miligrama de Equivalente de Ácido Gálico por $100 \mathrm{~g}$ da farinha).

\section{DETERMINAÇÃO DE TEOR DE FLAVANÓIDES}

Para determinação de flavonoides, em tubos de ensaio serão adicionados 2,0 mL da solução ( $5 \mathrm{~g}$ da farinha da casca de manga: $50 \mathrm{~mL}$ de água destilada) e $1,0 \mathrm{~mL}$ da solução metanólica de cloreto de alumínio $\left(\mathrm{AlCl}_{3}\right)$ a $5 \%(\mathrm{~m} / \mathrm{v})$ e 2,0 mL de metanol P.A e homegeneizada e posteriormente mantida em repouso em ausência de luz por um período de 30 min. Transcorrido esse tempo, absorbância da mistura reacional será medida em espectrofotômetro a $420 \mathrm{~nm}$. Os resultados serão expressos em mgEQ/100 g (miligramas de equivalente em Quercetina por 100 gramas da amostra).

\section{Resultados e Discussão}

A ACP dos tratamentos (FIGURA 2) mostrou que, o tratamento B apresentou resultados semelhantes ao tratamento $\mathrm{D}$ para todas as temperaturas de armazenamento, e maior semelhança para a temperatura de $7{ }^{\circ} \mathrm{C}$ em relação as demais. Ambos apresentaram boas características de conservação com o tempo de armazenamento, obtendo assim os tratamentos $\mathrm{B}$ e D a $7{ }^{\circ} \mathrm{C}$ como as melhores condições de conservação para a casca da Manga Tommy Atkins.

Figura 2. Análises dos componentes principais $(\mathrm{ACP})$ dos tratamentos em relação à temperatura do primeiro (a) e do último dia (b). (D0T0- tratamento controle dia zero; D0T1- tratamento A dia zero; D0T2- tratamento B dia zero; D0T3- tratamento C dia zero; D0T4- tratamento D dia zero; D4T0- 
tratamento controle $8^{\circ}$ dia; $\mathrm{D} 4 \mathrm{~T} 1$ - tratamento $\mathrm{A} 8^{\circ}$ dia; $\mathrm{D} 4 \mathrm{~T} 2$ - tratamento $\mathrm{B} 8^{\circ}$ dia; $\mathrm{D} 4 \mathrm{~T} 3$ - tratamento C $8^{\circ}$ dia; D4T4- tratamento $\mathrm{D} 8^{\circ}$ dia).

(a)

Biplot (axes F1 and F2: $\mathbf{5 1 . 9 8} \%$

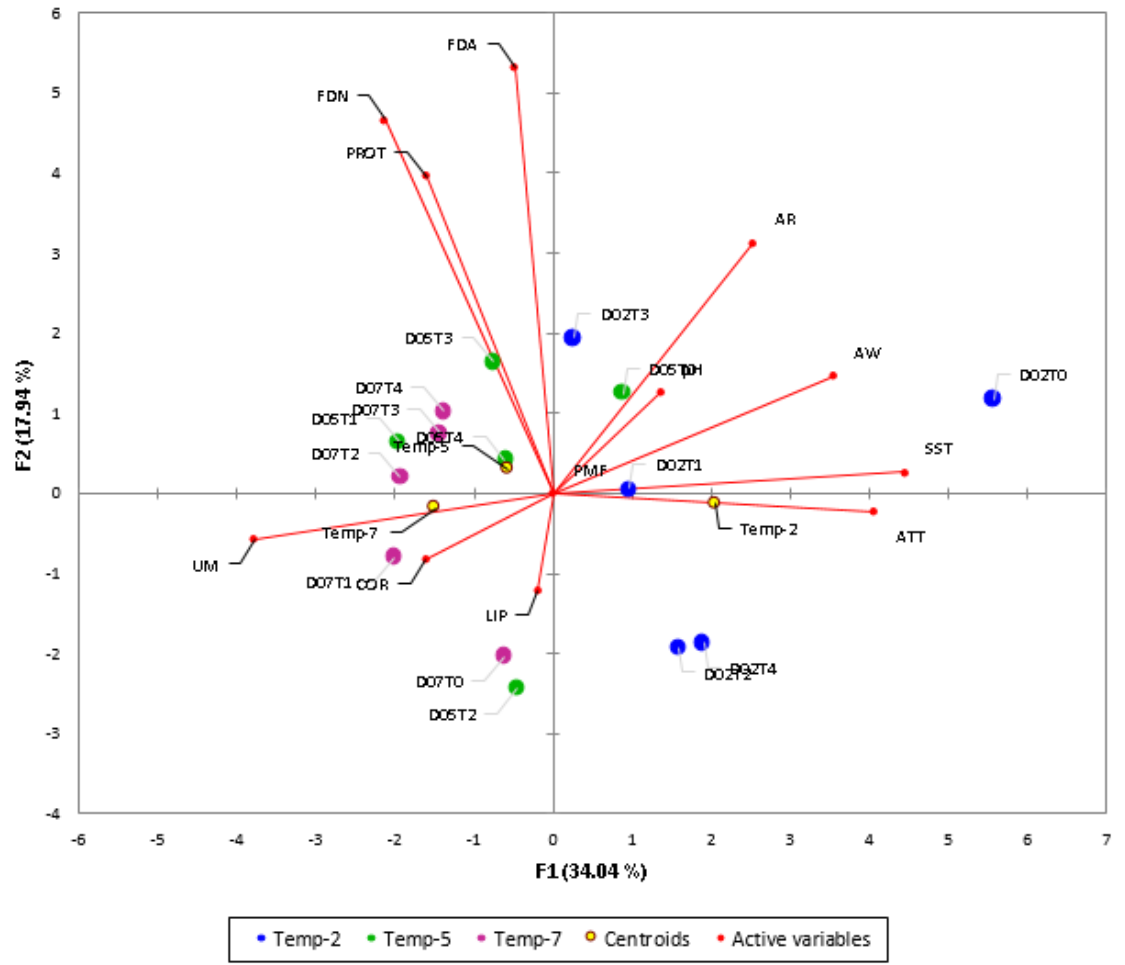

(b)

Biplot (axes F1 and F2: $\mathbf{4 3 . 5 9 \% )}$

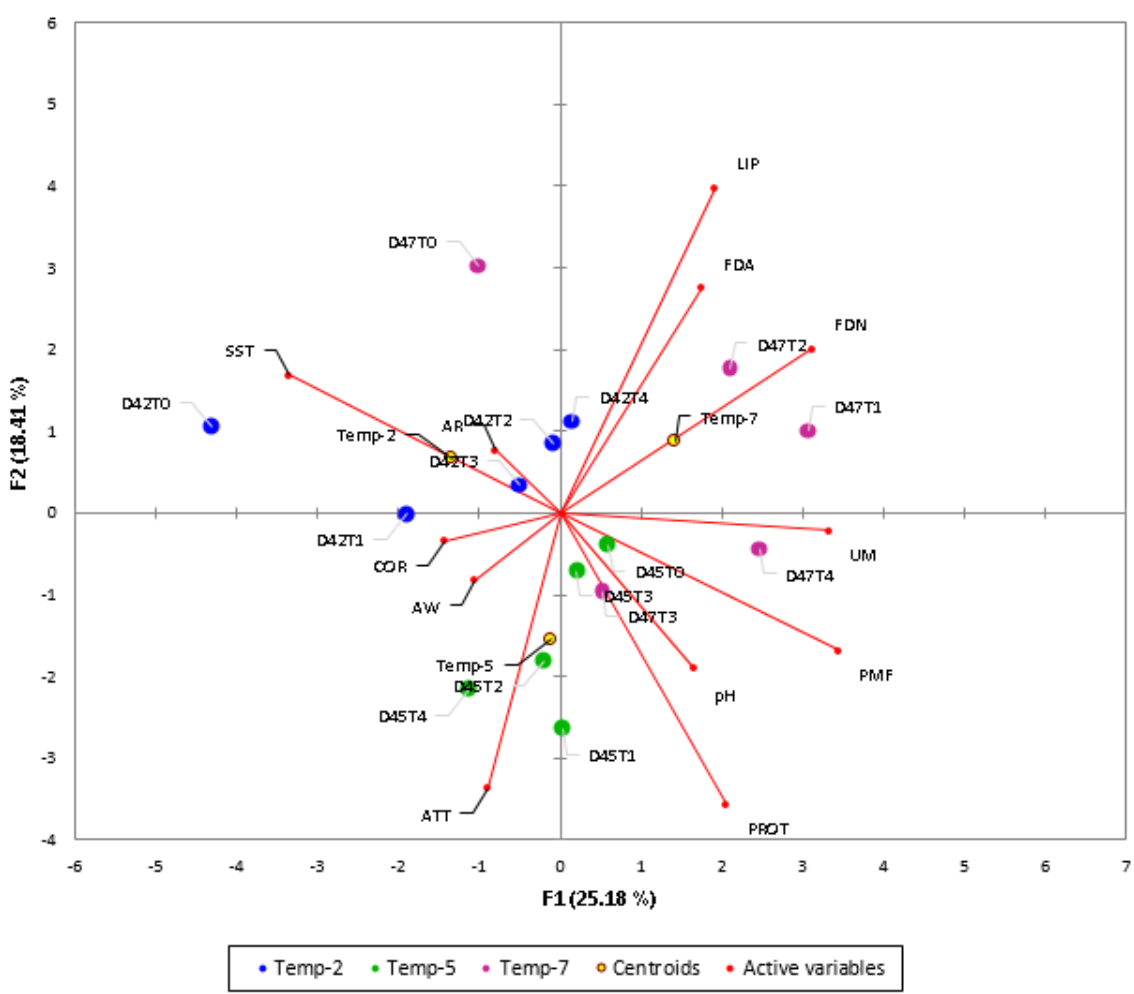

Fonte: Própria (2018). 
Quanto a farinha da casca de manga produzida a partir das melhores condições de tratamento (B e D) e na condição controle (FIGURA 3) apresentaram coloração amarronzada semelhante a encontrada por Moreno (2016) e Mendes (2013) tanto para sem tratamento a 7 ${ }^{\circ} \mathrm{C}$ quanto pra o tratamento B e D a $7{ }^{\circ} \mathrm{C}$.

Figura 3. Farinha da casca de manga CV Tommy Atkins sem tratamento e com tratamento B e D a temperatura de $7{ }^{\circ} \mathrm{C}$ com casca de 192 horas de armazenamento.

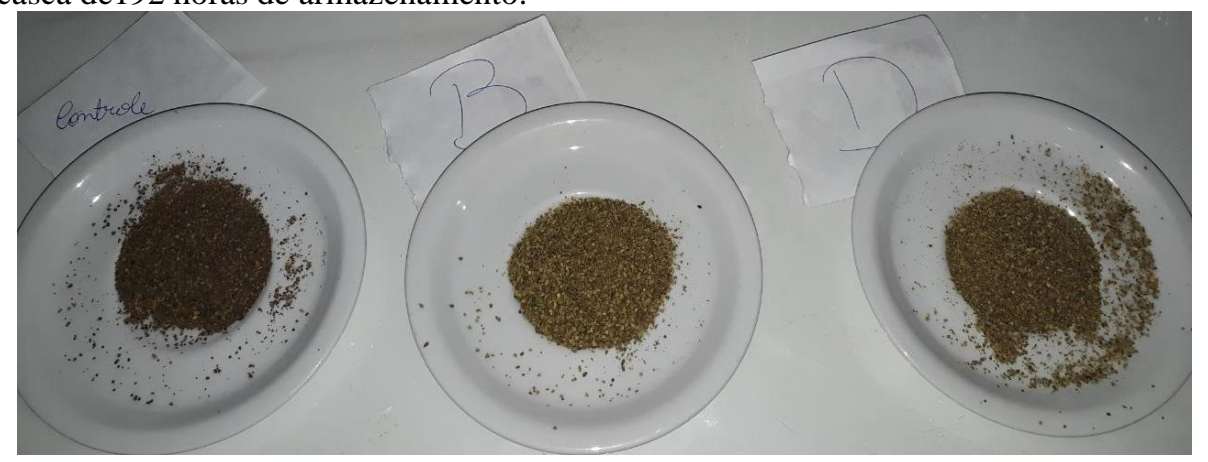

Fonte: Própria (2018).

A atividade antioxidante ABTS (TABELA 3) foi mais estável para as amostras que passaram por adição de inibidores, com destaque para o tratamento D, o que também foi verificado pela atividade antioxidante DPPH onde os tratamentos apresentaram diferenças significativas em relação a amostra controle, Rybka, Lima e Nassur (2018) durante a caracterização da farinha da casca de diferentes cultivares de manga obteve valor $(89,29 \%)$ para atividade antioxidante pelo método DPPH semelhante aos valores obtidos para os tratamentos B e D no presente trabalho. Ajila et al. (2010) em seu estudo sobre compostos bioativos e o potencial antioxidante de extratos provenientes da casca de manga, relatou uma alta atividade antioxidante e sugeriram, em função desta importante propriedade, o uso de casca de manga como nutracêutico e para alimentos funcionais.

Segundo Azevedo (2006) a maturação influencia de forma negativa na atividade antioxidante, o que pode justificar o menor teor de antioxidantes para a amostra controle visto que esta não recebeu adição de inibidores e com isso suas características podem ter sido afetadas durante os 8 dias de armazenamento, apresentando estádio de conservação inferior aos das amostras sob as condições dos tratamentos B e D, o maior teor de atividade antioxidante para os tratamentos B e D pode também ser atribuído a presença de ácido ascórbico nas soluções empregadas como conservantes, uma vez que este apresenta atividade antioxidante . 
Tabela 3. Avaliação da atividade antioxidante das farinhas da casca da manga CV Tommy Atkins controle, tratamento B e tratamento D, conservadas por 192 horas.

\begin{tabular}{l|l|l|l}
\hline Análises & \multicolumn{3}{|c}{ Resultado } \\
\hline Tratamentos & Controle & B & D \\
\hline Atividade antioxidante ABTS (\%) & $79,115 \pm 0,751^{\mathrm{C}}$ & $86,549 \pm 0,750^{\mathrm{B}}$ & $93,451 \pm 1,001^{\mathrm{A}}$ \\
Atividade antioxidante DPPH (\%) & $56,186 \pm 0,205^{\mathrm{B}}$ & $86,827 \pm 0,0103^{\mathrm{A}}$ & $67,249 \pm 5,146^{\mathrm{B}}$ \\
Compostos Fenólicos (mgEQ/100g) & $291,292 \pm 1,263^{\mathrm{B}}$ & $337,281 \pm 1,105^{\mathrm{A}}$ & $271,969 \pm 0,663^{\mathrm{C}}$ \\
& $37,639 \pm 1,155^{\mathrm{A}}$ & $39,306 \pm 0,575^{\mathrm{A}}$ & $39,125 \pm 0,832^{\mathrm{A}}$ \\
Flavonóides (mgEQ/100g) & & & \\
\hline
\end{tabular}

*As medias seguidas de letras distintas diferem entre si pelo teste de Tukey a 5\% de probabilidade

Fonte: Própria (2019)

A estrutura química dos fenólicos faz com que estes atuem como agentes redutores, reduzindo reações de oxidação através da doação de elétrons ou de hidrogênio aos radicais livres, ainda podem atuar reagindo com oxigênio singleite, fornecendo maior estabilidade aos alimentos (ARAUJO, 2012). AS farinhas apresentaram diferenças significativas (TABELA 3) entre si, o maior valor para compostos fenólicos foi atribuído a farinha de casca da manga proveniente do tratamento $\mathrm{B}$, valor este mais elevado que os valores obtidos por Melo \& Araújo (2011) que obtiveram para a manga Tommy Atkins um valor de 133,23 \pm 8,23 mg/100g, Os valores para o teor de fenólicos totais são susceptíveis a variação em função do clima, formas de cultivo, e solo onde foram cultivadas as mangas (CALDAS E SANTOS, 2018).

Variações na concentração de flavonoides nas frutas pode ser influenciada por fatores como sazonalidade, incidência de radiação UV, clima, composição do solo, preparo e processamento do alimento (BRAMONT et al., 2018). Não foram verificadas diferenças significativas para os tratamentos em relação ao teor de flavonoides (TABELA 3), sendo o maior valor atribuído para farinha proveniente do tratamento $\mathrm{B}$, o que é muito interessante uma vez que atividades anti-inflamatórias e cicatrizantes são amplamente relacionadas com a presença de taninos e flavonoides em alimentos (ARAUJO, 2008).

\section{Conclusões}

Através do presente estudo foi verificado que as farinhas da casca da manga apresentaram potencial antioxidante, demonstrando o potencial de aplicabilidade destas, 
observa-se os maiores teores de DPPH, compostos fenólicos e flavonoides para farinha da casca da manga proveniente do tratamento $\mathrm{B}$, sendo verificado que o emprego dos tratamentos com inibidores de reação (ácido ascórbico e cloreto de cálcio) indicando maior estabilidade de armazenamento e preservação de compostos.

\section{Referências}

AJILA, C. M., M. Aalami, K. Leelavathi, U. J. S. Prasada Rao. Mango peel powder: A potential source of antioxidant and dietary fi ber in macaroni preparations. Innovative Food Science \& Emerging Technologies, v.11, n.1, p.219-224, Out, 2010.

ARAUJO, C. R. de. Cascas liofilizadas de manga tommy Atkins: teor de fitoquimicos bioativos e potencial antioxidante. Tese de mestrado (131). Universidade Federal Rural de Pernambuco, p. 24-38. Recife-PE, 2012.

ARAUJO, T. A. de S. Taninos e flavonoides em plantas medicinais da caatinga: um estudo de etnobotânica quantitativa. Tese de mestrado (71). Universidade Federal de Pernambuco, p. 21-31. Recife-PE, 2008.

AZEVEDO, A. C. S. Estudo das enzimas oxidativas e presença de compostos bioativos em mangas (Mangifera indica L.) produzidas no Brasil. Tese de doutorado em Ciência de Alimentos, (196). Universidade estadual de campinas faculdade de engenharia de alimentos departamento de ciência de alimentos, p. 128-138. Campinas - SP, 2006.

BRAMONT, W. B.; I. L. LEAL; M. A. UMSZA-GUEZ; A. S. GUEDES; S. C. O. ALVES; J. H. O. REIS; J. D. V. BARBOSA; B. A. S. MACHADO. Comparação da Composição Centesimal, Mineral e Fitoquímica de Polpas e Cascas de Dez Diferentes Frutas. Revista virual de química, v.10, n.4. Salvador-BA, Jul., 2018.

BRASIL. Agência Nacional de Vigilância Sanitária. Resolução RDC n. 263, de 22 de setembro de 2005. Regulamento técnico para produtos de cereais, amidos, farinhas e farelos. Set., 2005.

CALDAS J da C.; W. N. L. dos SANTOS. DETERMINAÇÃO DE COMPOSTOS BIOATIVOS EM CASCA DE MANGA (Mangifera indica L.) POR CROMATROGRAFIA LÍQUIDA DE ALTA EFICIÊNCIA - CLAE. Anais do evento. 70ª Reunião Anual da SBPC-UFAL, v.70 n.1; p. 1-2, Maceió, Jul., 2018.

DETMANN, E; M. A.de SOUZA; S.de C.VALADARES FILHO; A. C. de QUEIROZ; T. T. BERCHIELLI; E. de O. S. SALIBA; L. da S. CABRAL; D. dos S. PINA; M. M. LADEIRA; J.A.G. AZEVEDO. Métodos para análise de alimentos. Instituto nacional de ciência e tecnologia de ciência animal. $1^{\circ}$ ed. 2012.

EL-FAHAM, S. Y.; M. S. ASHOUR MOHSEN; A. M., SHARAF; A. A. ZAKY. Utilization of Mango Peels as a Source of Polyphenolic Antioxidants. Current Science International, v.5, n.4, p.529 - 542. Dez., 2016. 
INSTITUTO ADOLFO LUTZ. Normas analíticas do Instituto Adolfo Lutz. Métodos físico-químicos para análise de alimentos. São Paulo: IMESP, 2008.

LEMOS, D. M.; S. F.DA SILVA; J. C. B. DE LIMA; F. B. DA SILVA; E. P. DE SOUSA. Parâmetros químicos, físicos e físico-químicos de resíduos da manga. Revista Verde de Agroecologia e Desenvolvimento Sustentável, v.8, n.2, p.01 - 03. Mossoró - RN, Jun., 2013.

LI, Y., B. JIANG, T. ZHANG, W. MU, J. LIU. Antioxidant and free radical scavenging activities of chickpea protein hydrolysate (CPH). Food Chemistry, 2008.

MALDONADE, I. R.; P. G. B. CARVALHO; N.A. FERREIRA. Protocolo para determinação de açúcares totais em hortaliças pelo método de DNS. EMBRAPA. $1^{\circ}$ ED, Mar., 2013.

MASUD PARVEZ, G. M.; A. MOSADDIK. Evaluation of anticancer property of mango peel and flesh after formalin treatment. JPHYTO, V.5, N.3P. 112-116, Jun., 2016.

MELO, E. A.; C. R. ARAÚJO. Mangas das variedades espada, rosa e tommy atkins: compostos bioativos e potencial antioxidante. Semina: Ciências Agrárias, Londrina, v. 32, n. 4, p. 1451-1460, out./dez., 2011.

MENDES, B. de A. B. Obtenção, Caracterização e aplicação de farinha das cascas de abacaxi e de manga. Tese de Mestrado (74). Universidade Estadual do Sudoeste da Bahia (UESB), p. 45-47. Itapetinga -Bahia, 2013.

MIGUEL, A. C. A. Uso de película comestível, cloreto de cálcio e ácido ascórbico para a conservação do melão 'amarelo' minimamente processado. Tese de mestrado (196). Universidade de são Paulo escola superior de agricultura "Luiz Queiroz", p. 17-33. Piracicaba, 2008.

MORAIS, M. L.; A. C. R. SILVA; C. R. R. ARAÚJO; E. A. ESTEVES; N. A. V. DESSIMONI-PINTO Determinação do potencial antioxidante in vitro de frutos do cerrado brasileiro. Revista Brasileira. Fruticultura, Jaboticabal - SP, v. 35, n. 2, p. 355-360, Jun. 2013.

MORENO, J. de S. Obtenção, caracterização e aplicação de farinha de resíduos de frutas em cookies. Tese de mestrado (82). Universidade estadual do sudoeste da Bahia, p. 15-22. Itapetinga-BA, 2016.

OLIVAS, G. I.; D. S. MATTINSON; G. V. BARBOSA-CÁNOVAS. Alginate coatings for preservation of minimally processed 'Gala' apples. Postharvest Biology and Technology, 45 p.89-96. 2007.

OLIVEIRA, I. R. N. DE. Antocianinas extraídas de capim-gordura (Melinis minutiflora): atividade antioxidante, microencapsulamento por atomização e estabilidade. Tese de mestrado. Programa de Pós-Graduação em Ciência e Tecnologia de Alimentos, Universidade Federal de Viçosa (UFV), Viçosa, 2011. 
PADILHA, T.; C. BASSO. Biscoitos com resíduo de manga, maracujá e jabuticaba. Disciplinarum Scientia. Série: Ciências da Saúde, v.16, n.1, p.79-88. Santa Maria, Mai., 2015.

REINOSO, A. C. L.; R. R. DE SOUZA; M. A. G. CARNELOSSI; A. M. DA CONCEIÇÃO C. C. DE S. BERY; J. K.DE OLIVEIRA. Efeitos das temperaturas na conservação dos minerais em farinhas de cascas de mangas. Interfaces Científicas - Saúde e Ambiente, v.5, n.2, p. $25-32$. Aracaju, fev., 2017.

RYBKA, A. C. P., A. de S. LIMA, R. de C. M. R. NASSUR. Caracterização da farinha da casca de diferentes cultivares de manga. Anais do evento. ENCICLOPEDIA BIOSFERA. Centro Científico Conhecer, v.15 n.27; p. 12, Goiânia, 2018.

SINGLETON, V. L.; ROSSI, J. A. Colorimetry of Total Phenolics with PhosphomolybdicPhosphotungstic Acid Reagents. Am. J. Enol. Vitic., v. 16, p. 144-158, 1965.

SOUSA, S. B.; CARVAlHO, A. V.; MATTIETTO, R. A.; OlIVEIRA, M. S. Compostos fenólicos e atividade antioxidante de frutos de bacaba (oenocarpus spp.). XXV Congresso Brasileiro de Ciência e Tecnologia de Alimentos, 24 a 27 de Out., 2016.

SUCUPIRA, N. R.; SILVA, A. B.; PEREIRA, G.; COSTA, J. N. Métodos Para Determinação da Atividade Antioxidante de Frutos. UNOPAR Cient Ciênc Biol Saúde; v.14 n.4, p. 263-269, 2012.

SWAIN, T.; HILLS, W.E. The phenolic constituents of Punnus domestica . The quantitative analysis of phenolic constituents. Journal of the Science of Food and Agriculture, London, v.19, p. 63-68, 1959.

TSENG TH, KAO ES, CHU CY, CHOU FP, LIN HW, WANG CJ. Protective effects of dried flower extracts of Hibiscus sabdariffa L. Against oxidative stress in rat primary hepatocytes. Food and Chemical Toxicology, v.35, n.2; p.59-1164, Taiwan, 2006.

VIEIRA, L.M.; SOUSA, M.S.B.; MANCINI-FILHO, J.; LIMA, A. Fenólicos totais e capacidade antioxidante in vitro de polpas de frutos tropicais. Revista Brasileira de Fruticultura, v.33, n.3, p. 888-897, 2011.

WOISKY, R. G.; SALATINO, A.. Analysis of propolis: some parameters and procedures for chemical quality control. Journal of Apicultural Research, v.37, n 2, p. 99-105 1998

YU-GE LIU, X. Z.; M. FEI-YUE; F. QIONG. The antioxidant activitives of mango peel among different cultivars. $3^{\circ}$ International Conference on Energy Materials and Environment Engineering. V. 61 N. 1, 2017. 